San Jose State University

SJSU ScholarWorks

Faculty Publications

Physics and Astronomy

$1-1-2012$

\title{
Dwarfs gobbling dwarfs: a stellar tidal stream around NGC 4449 and hierarchical galaxy formation on small scales
}

\author{
D. Mart'inez-Delgado \\ Max-Planck-Institut für Astronomy \\ Aaron J. Romanowsky \\ San Jose State University, aaron.romanowsky@sjsu.edu \\ R. J. Gabany \\ Black Bird Observatory \\ F. Annibali \\ Osservatorio Astronomico di Bologna \\ J. A. Arnold \\ University of California, Santa Cruz
}

See next page for additional authors

Follow this and additional works at: https://scholarworks.sjsu.edu/physics_astron_pub

Part of the Astrophysics and Astronomy Commons

\section{Recommended Citation}

D. Mart'inez-Delgado, Aaron J. Romanowsky, R. J. Gabany, F. Annibali, J. A. Arnold, J. Fliri, S. Zibetti, R. P. van der Marel, H. W. Rix, T. S. Chonis, J. A. Carballo-Bello, A. Aloisi, A. V. Macci`o, J. Gallego-Laborda, J. P. Brodie, and M. R. Merrifield. "Dwarfs gobbling dwarfs: a stellar tidal stream around NGC 4449 and hierarchical galaxy formation on small scales" Astrophysical Journal Letters (2012). https://doi.org/ 10.1088/2041-8205/748/2/L24

This Article is brought to you for free and open access by the Physics and Astronomy at SJSU ScholarWorks. It has been accepted for inclusion in Faculty Publications by an authorized administrator of SJSU ScholarWorks. For more information, please contact scholarworks@sjsu.edu. 


\section{Authors}

D. Mart'inez-Delgado, Aaron J. Romanowsky, R. J. Gabany, F. Annibali, J. A. Arnold, J. Fliri, S. Zibetti, R. P. van der Marel, H. W. Rix, T. S. Chonis, J. A. Carballo-Bello, A. Aloisi, A. V. Macci’o, J. Gallego-Laborda, J. P. Brodie, and M. R. Merrifield 


\title{
DWARFS GOBBLING DWARFS: A STELLAR TIDAL STREAM AROUND NGC 4449 AND HIERARCHICAL GALAXY FORMATION ON SMALL SCALES
}

\author{
David Martínez-Delgado ${ }^{1,13}$, Aaron J. Romanowsky ${ }^{2}$, R. Jay Gabany ${ }^{3}$, Francesca Annibali ${ }^{4}$, Jacob A. Arnold ${ }^{2}$, \\ Jürgen Fliri ${ }^{5,6}{ }^{,}$Stefano Zibetti $^{7}$, Roeland P. van der Marel ${ }^{8}$, Hans-Walter Rix ${ }^{1}$, Taylor S. Chonis $^{9}$, \\ Julio A. Carballo-Bello ${ }^{10}$, Alessandra Aloisi ${ }^{8}$, Andrea V. Macciò ${ }^{1}$, J. Gallego-Laborda ${ }^{11}$, \\ JEAN P. BRodie ${ }^{2}$, AND Michael R. MERRIFIELD ${ }^{12}$ \\ ${ }^{1}$ Max-Planck-Institut für Astronomy, Heidelberg, Germany \\ ${ }^{2}$ UCO/Lick Observatory, University of California, Santa Cruz, CA 95064, USA \\ ${ }^{3}$ Black Bird Observatory, Mayhill, New Mexico, USA \\ ${ }^{4}$ Osservatorio Astronomico di Bologna, INAF, Via Ranzani 1, I-40127 Bologna, Italy \\ ${ }^{5}$ LERMA, CNRS UMR 8112, Observatoire de Paris, 61 Avenue de l'Observatoire, F-75014 Paris, France \\ ${ }^{6}$ GEPI, CNRS UMR 8111, Observatoire de Paris, 5 Place Jules Janssen, F-92195 Meudon, France \\ ${ }^{7}$ Dark Cosmology Centre, Niels Bohr Institute-University of Copenhagen, Juliane Maries Vej 30, DK-2100 Copenhagen, Denmark \\ ${ }^{8}$ Space Telescope Science Institute, 3700 San Martin Drive, Baltimore, MD 21218, USA \\ ${ }^{9}$ Department of Astronomy, University of Texas at Austin, Texas, USA \\ ${ }^{10}$ Instituto de Astrofisica de Canarias, Tenerife, Spain \\ ${ }^{11}$ Fosca Nit Observatory, Montsec Astronomical Park, Ager, Spain \\ ${ }^{12}$ School of Physics and Astronomy, University of Nottingham, University Park, Nottingham NG7 2RD, UK \\ Received 2011 December 9; accepted 2012 January 31; published 2012 March 15
}

\begin{abstract}
A candidate diffuse stellar substructure was previously reported in the halo of the nearby dwarf starburst galaxy NGC 4449 by Karachentsev et al. We map and analyze this feature using a unique combination of deep integrated-light images from the BlackBird $0.5 \mathrm{~m}$ telescope, and high-resolution wide-field images from the $8 \mathrm{~m}$ Subaru Telescope, which resolve the nebulosity into a stream of red giant branch stars, and confirm its physical association with NGC 4449. The properties of the stream imply a massive dwarf spheroidal progenitor, which after complete disruption will deposit an amount of stellar mass that is comparable to the existing stellar halo of the main galaxy. The stellar mass ratio between the two galaxies is $\sim 1: 50$, while the indirectly measured dynamical mass ratio, when including dark matter, may be $\sim 1: 10-1: 5$. This system may thus represent a "stealth" merger, where an infalling satellite galaxy is nearly undetectable by conventional means, yet has a substantial dynamical influence on its host galaxy. This singular discovery also suggests that satellite accretion can play a significant role in building up the stellar halos of low-mass galaxies, and possibly in triggering their starbursts.
\end{abstract}

Key words: galaxies: dwarf - galaxies: evolution - galaxies: interactions - galaxies: structure

\section{INTRODUCTION}

A fundamental characteristic of the modern cold dark matter $(\Lambda \mathrm{CDM})$ cosmology (Mo et al. 2010) is that galaxies assemble hierarchically under the influence of gravity, continually accreting smaller DM halos up until the present day. If those halos contain stars, then they will be visible as satellites around their host galaxies, eventually disrupting through tidal forces into distinct streams and shells before phase mixing into obscurity (Cooper et al. 2010). This picture seems to explain the existence of satellites and substructures observed around massive galaxies (Arp 1966; Schweizer \& Seitzer 1988; McConnachie et al. 2009; Martínez-Delgado et al. 2010). However, quantitative confirmation of this aspect of $\Lambda C D M$ has been more elusive, with lingering doubts provoked by small-scale substructure observations (Lovell et al. 2012; Boylan-Kolchin et al. 2012; Ferrero et al. 2012).

There has been relatively little work on substructure and merging in the halos around low-mass, "dwarf" galaxies. Many cases of extended stellar halos around dwarfs have been identified observationally (Stinson et al. 2009), but it is not clear if these stars were accreted, or formed in situ. Star formation in dwarfs is thought to occur in stochastic episodes (Tolstoy et al. 2009; Weisz et al. 2011), which could be triggered by accretion events.

\footnotetext{
$\overline{13}$ Alexander von Humboldt Fellow for Advanced Research.
}

An iconic galaxy in this context is NGC 4449, a dwarf irregular in the field that has been studied intensively as one of the strongest galaxy-wide starbursts in the nearby universe. Its absolute magnitude of $M_{V}=-18.6$ makes it a Large Magellanic Cloud (LMC) analogue (and not formally a dwarf by some definitions), but with a much higher star formation rate. It is strongly suspected to have recently interacted with another galaxy based on various signatures including peculiar kinematics in its cold gas and H II regions (Hartmann et al. 1986; Hunter et al. 1998), but the nature of this interaction is unknown.

An elongated dwarf galaxy or stream candidate near NGC 4449 was first noticed by Karachentsev et al. (2007) from Digitized Sky Survey (POSS-II) plates (object d1228+4358), and is visible in the Sloan Digital Sky Survey (SDSS). ${ }^{14}$ Here we present deep, wide-field optical imaging that supplies the definitive detection of this ongoing accretion event involving a smaller galaxy, leading to interesting implications about the evolution of this system and of dwarf galaxies in general.

\section{OBSERVATIONS AND DATA REDUCTION}

Our observations of NGC 4449 and its surroundings consist of two main components. The first is imaging from a small robotic telescope to confirm the presence of a low-surfacebrightness substructure and provide its basic characteristics

\footnotetext{
14 http://www.sdss.org/
} 

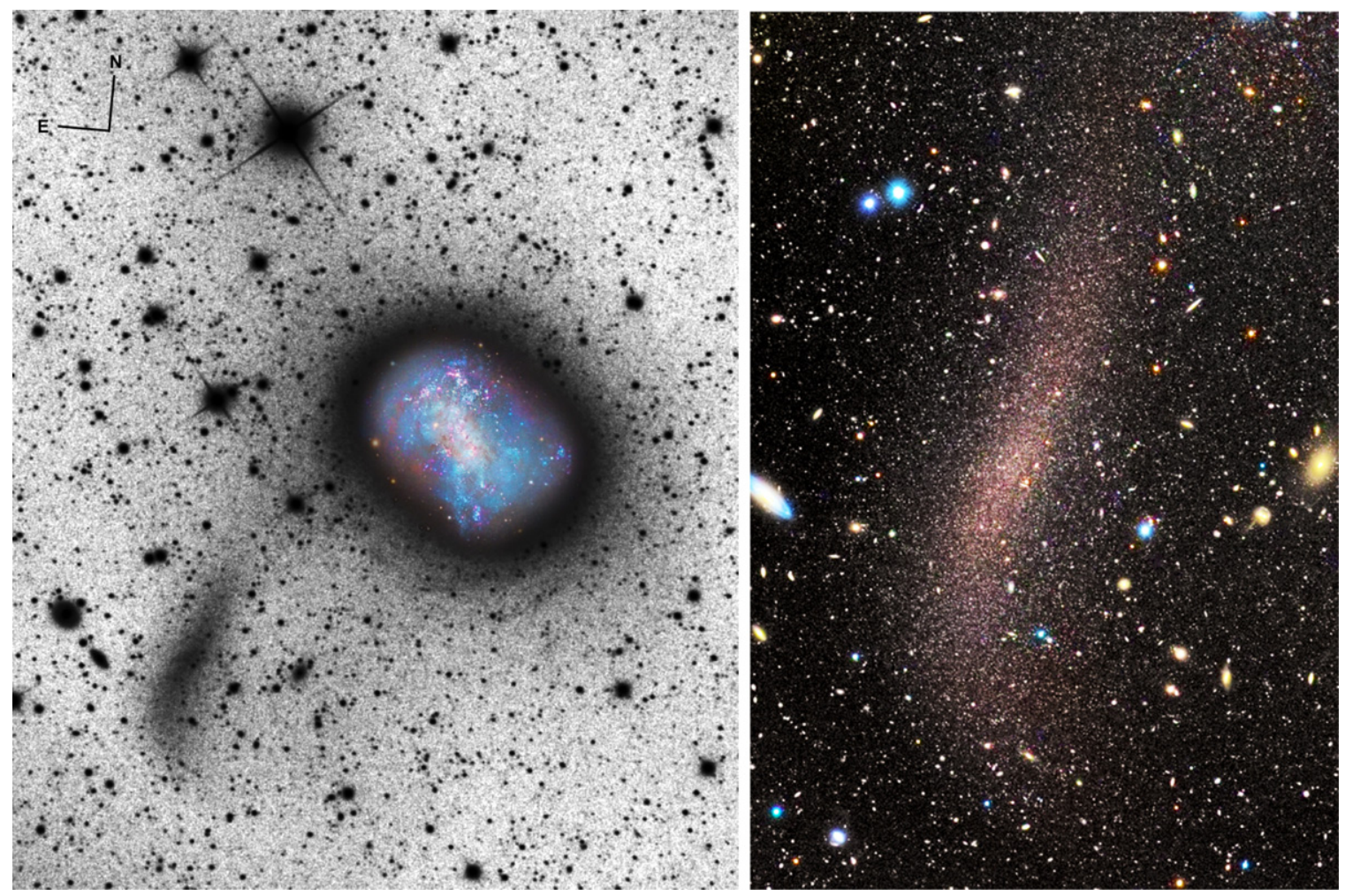

Figure 1. NGC 4449 and its halo stream. Left: image from BBRO, showing a 19'.0 × $24.5(21 \times 27 \mathrm{kpc})$ field. Right: $5.5 \times 8.6(6 \times 9.5 \mathrm{kpc})$ subsection of the Subaru/Suprime-Cam data, showing the stream resolved into stars. In both panels, shallower BBRO exposures in red/green/blue filters provide indicative colors.

(similar techniques were used with larger galaxies in MartínezDelgado et al. 2008, 2010). The second is follow-up imaging with the Subaru Telescope to map out the resolved stellar populations.

We obtained very deep images with the $f / 8.3$ RitcheyChretien $0.5 \mathrm{~m}$ telescope of the BlackBird Remote Observatory $(\mathrm{BBRO})^{15}$ during different dark-sky observing runs over the periods 2010 April 13 through June 10 and 2011 January 13 through 28 (UT). We used a 16 mega-pixel Apogee Imaging Systems U16M CCD camera, with 31 '.3 $\times 31$ '.3 field-of-view and 0.46 arcsec pixel $^{-1}$ plate scale. We acquired $18 \mathrm{hr}$ of imaging data in half-hour sub-exposures, using a non-infrared clear luminance ( $\lambda=3500-8500 \AA)$ Astrodon E-series filter. Each sub-exposure was reduced following standard procedures for dark-subtraction, bias-correction, and flat-fielding (MartínezDelgado et al. 2009).

The resulting image was calibrated photometrically to SDSS using the brighter regions of NGC 4449 (see Martínez-Delgado et al. 2010). The final image has $5 \sigma g$-band surface-brightness detection limits from 26.4 to $27.5 \mathrm{mag} \operatorname{arcsec}^{-2}$ for seeinglimited and large-scale diffuse features, respectively.

We subsequently obtained images from the $8.2 \mathrm{~m}$ Subaru Telescope and the Suprime-Cam wide-field imager $\left(34^{\prime} \times\right.$ $27^{\prime}$ field-of-view, 0'202 pixel scale; Miyazaki et al. 2002) on 2011 January 5 (UT). Conditions were photometric, and we took dithered exposures in $r^{\prime}$ and $i^{\prime}$ bands, with total exposure times of $225 \mathrm{~s}$ per filter. We reduced the data using a modified SDFRED pipeline (Ouchi et al. 2004), including bias subtraction, flat-fielding, and distortion correction. Each frame was re-projected to a common astrometric coordinate system

15 BBRO was originally situated in the Sacramento Mountains (New Mexico, USA), and later moved to the Sierra Nevada Mountains (California, USA). followed by background rectification and image co-addition using Montage. ${ }^{16}$

The exquisite image quality ( $\left.\sim 0^{\prime \prime} 5 \mathrm{FWHM}\right)$ allows us to resolve individual stars in the outer regions of NGC 4449. We carried out point-spread-function photometry using DAOPHOT II/ALLSTARS (Stetson 1987) and identified stars as objects with sharpness parameter $|S|<1.0$. We calibrated the photometry based on two central images from the Hubble Space Telescope Advanced Camera for Surveys (HST/ACS; Annibali et al. 2008, hereafter A+08).

The ACS photometry was originally in F555W and F814W, and recalibrated to Johnson-Cousins $V I(\mathrm{~A}+08)$. We used fairly bright, red stars in common between the data sets to derive linear transformation equations from $r^{\prime} i^{\prime}$ instrumental magnitudes to $V I$, including foreground extinction corrections of $E(B-V)=$ 0.019 (Schlegel et al. 1998). Our final star catalog has statistical internal errors in $V-I$ color of $\sim 0.11 \mathrm{mag}$ and $\sim 0.14$ mag at $V$ $=25$ and $V=26$, respectively.

\section{STREAM MORPHOLOGY}

Figure 1 (left) shows a BBRO image subsection, where with an adopted distance of $3.82 \mathrm{Mpc}(\mathrm{A}+08)$, 1' corresponds to $1.1 \mathrm{kpc}$. Clearly visible near the minor axis of NGC 4449, $\sim 10 \mathrm{kpc}$ to the southeast, is a very elongated, S-shaped feature of dimensions $\sim 1.5 \times 7 \mathrm{kpc}$, which we designate "the stream." The stream's position does not overlap with any of the complex H II-gas features surrounding NGC 4449 (Hunter et al. 1998). Also, it is on the opposite side of the galaxy with respect to an interesting star cluster that may be linked to another past accretion event (Annibali et al. 2012). The main galaxy's

\footnotetext{
16 http://montage.ipac.caltech.edu/
} 


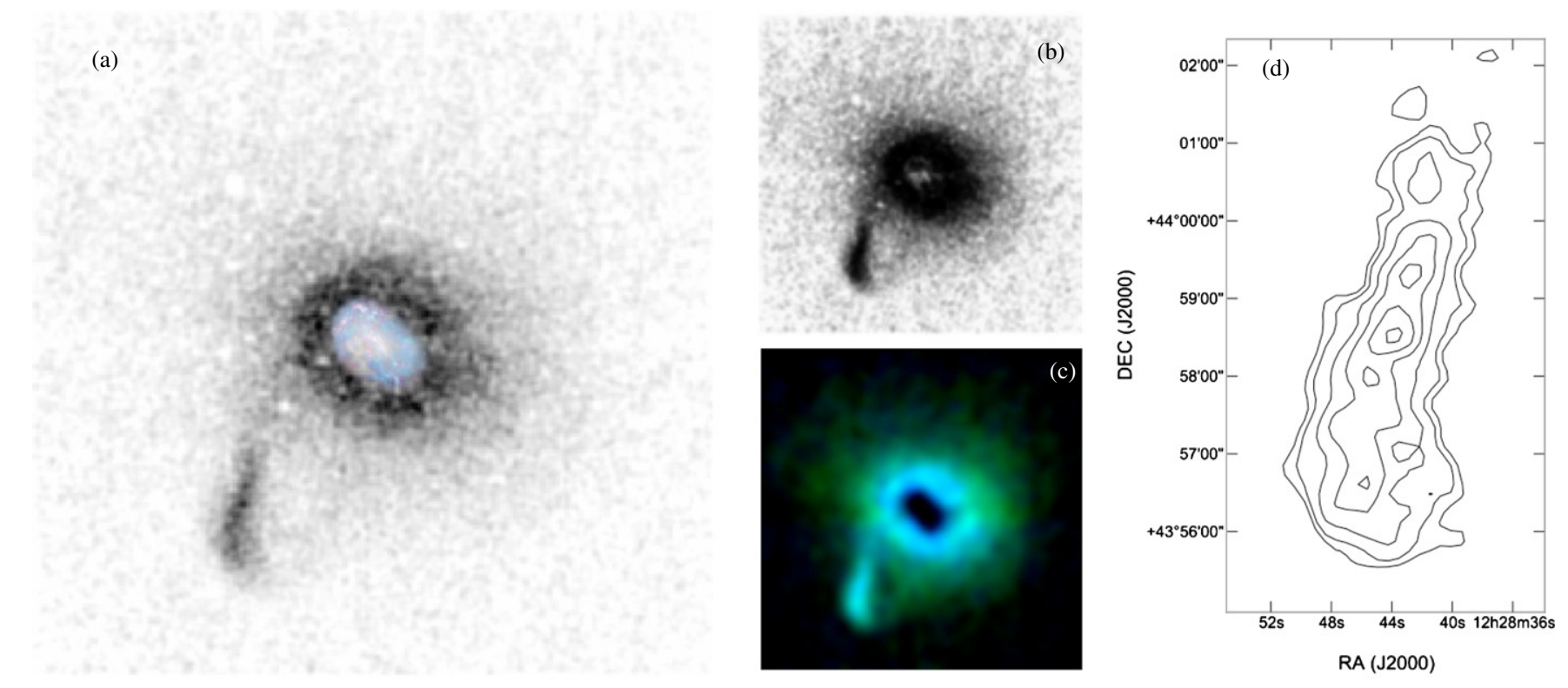

Figure 2. Stellar-density maps of NGC 4449 and its stream, based on RGB counts. (a) $30^{\prime} \times 30^{\prime}(33 \times 33 \mathrm{kpc})$ image with linear scaling. A compact density enhancement within the stream may be the progenitor galaxy's remnant nucleus. (b) The same data with less dynamic range. The stream shows a loop-like structure curving back toward the main galaxy, which in turn also shows a shell-like overdensity in its halo toward the southwest, reaching similar projected galactocentric radii as the stream. (c) Composite map of the stream, color-coded blue for bluer RGB colors, and green for redder colors (see main text). The RGBs in the stream's main part have similar average colors to the main galaxy's inner halo stars, with redder populations apparent on the outskirts of both the halo and stream. (d) Star-count contour map of the stream, with contour levels corresponding to 5-10 stars per $50 \operatorname{arcsec}^{2}$ bin, in intervals of $1 \mathrm{star}$ per bin.

existing stellar halo is also apparent, including shell-like features in the southwest previously noticed by Hunter et al. (1999).

The right-hand panel shows a Suprime-Cam zoom-in of the stream. It is clearly resolved into stars and has the general appearance of a dwarf spheroidal (dSph) galaxy that is elongated by tides. This classification (defined by a spheroidal morphology, stellar mass $M_{\star}<10^{8} M_{\odot}$, and the absence of star formation and cold gas) is also supported by the $\mathrm{H}$ I non-detection of Huchtmeier et al. (2009).

To complement these integrated surface-brightness maps, we construct stellar-density maps around NGC 4449 using individual red giant branch (RGB) stars down to $g^{\prime}=25.8$ from Suprime-Cam. We discuss the RGB selection and modeling later, but in general these stars trace a population older than 1 Gyr. We define a grid across the image with $130 \times 130 \mathrm{pc}^{2}$ bins and count the number of stars in each bin, subsequently applying a Gaussian smoothing kernel with $\sigma_{\text {smooth }}=130 \mathrm{pc}$. There are typically seven to nine stars per bin in the stream.

Figure 2(a) shows the resulting Subaru-based stellar-density map, where the overall stream morphology is indistinguishable from the BBRO integrated-light results. Since there is no evidence for resolved young stars (see next section), we infer that the visible light is dominated by RGB stars and hence by a population older than $1 \mathrm{Gyr}$.

Panel (b) shows a higher-contrast version of the same map, demonstrating that the main galaxy's preexisting stellar halo extends out to at least $\sim 10 \mathrm{kpc}$. We can furthermore discern a very faint feature that seems to extend the stream's angular path in a loop-like structure. This loop is also apparent in the BBRO image, and we infer that the stream is stretched out over at least half its orbit, with the projected turning point at a galactocentric radius of $13 \mathrm{kpc}$.

We next wish to locate the disrupting satellite galaxy's original center or nucleus. In both the BBRO and Subaru images there is a density enhancement near the mid-point of the "S," as would be expected if the "arms" are leading and trailing tidal tails around a still marginally bound, or just disrupting, main body. We construct a stellar-density contour map for the stream region, using RGB stars and $\sigma_{\text {smooth }}=270 \mathrm{pc}$. The central stellar clump is visible in Figure 2(d), with a position slightly offset from the stream's main ridgeline $\left(\alpha_{\mathrm{J} 2000}=122843.32, \delta_{\mathrm{J} 2000}=\right.$ +435830.0 ; positional uncertainty $\left.\sim 6^{\prime \prime}\right)$. An off-center nucleus is also seen in a tidally disrupting Milky Way dSph, Ursa Major (Martínez-Delgado et al. 2001).

\section{STELLAR POPULATIONS}

Figure 3 shows the color-magnitude diagram (CMD) for point sources in the stream region. The RGB stars are the dominant feature, along with a few brighter, redder stars that may be oxygen- or carbon-rich thermally pulsating asymptotic giant branch stars from an intermediate-age or old population (Marigo et al. 2003). We find no blue stars that would trace recent star formation.

The detection of the tip of the RGB (TRGB) permits a distance estimate. Using techniques from A+08 and Cioni et al. (2000), we find a TRGB magnitude of $I_{\mathrm{TRGB}}=24.06 \pm 0.04$ (random) \pm 0.08 (systematic). The random error was estimated using bootstrapping techniques; the systematic error is dominated by the magnitude-transformation uncertainties. The main body of NGC 4449 was found by $\mathrm{A}+08$ to have $I_{\mathrm{TRGB}}=24.00 \pm$ 0.01 (random) \pm 0.04 (systematic). Thus, the stream is at the same distance as the main body, to within $\sim 180 \mathrm{kpc}$, and we conclude that there is a physical association rather than a chance superposition.

Although the RGB is affected by the well-known age-metallicity degeneracy, this feature can still be used to constrain the properties of stars older than $\sim 1$ Gyr. In Figure 3, we overplot the Padua isochrones (Girardi et al. 2002) for ages of 2, 4, and $10 \mathrm{Gyr}$, for both $Z=0.004$ and $Z=0.001$. For $Z=0.004$ the $2-4$ Gyr isochrones trace the data reasonably well. The $Z=0.001$ models appear significantly 


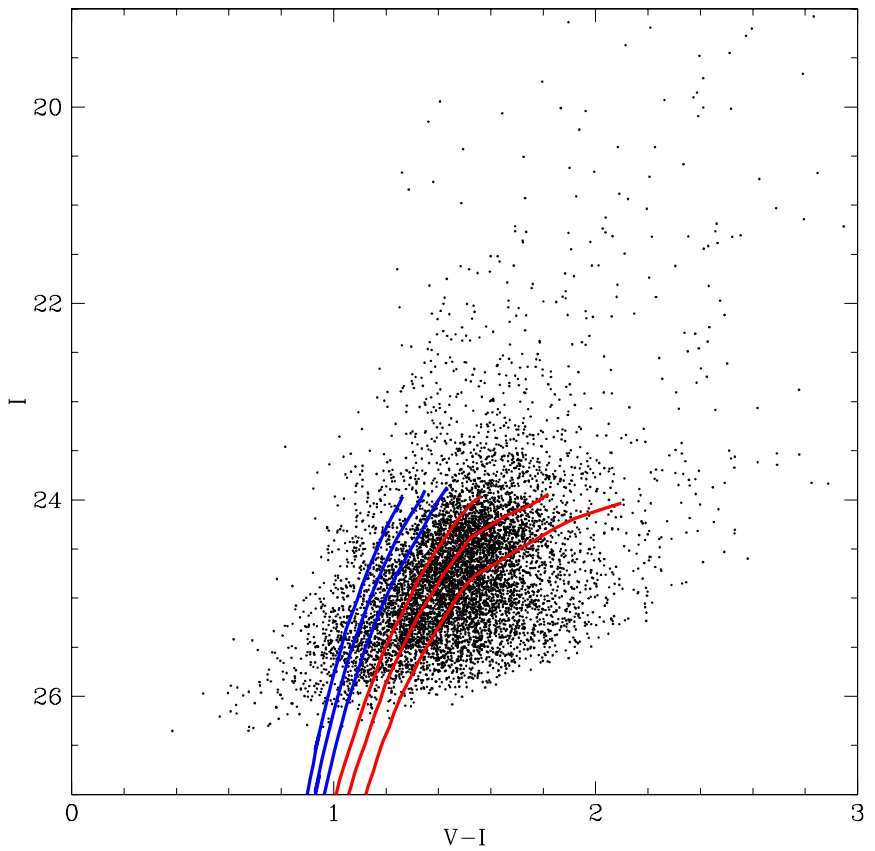

Figure 3. Color-magnitude diagram of the stream region, centered at $\left(\alpha_{\mathrm{J} 2000}=\right.$ $\left.122843, \delta_{\mathrm{J} 2000}=+435830.0\right)$, with a $250^{\prime \prime} \times 540^{\prime \prime}(4.6 \times 10.0 \mathrm{kpc})$ field of view, using $r^{\prime} i^{\prime}$ photometry transformed to $V I$. RGB isochrones are overlaid for metallicities $Z=0.001$ (blue curves) and $Z=0.004$ (red curves). For each $Z$, the isochrones show ages of 2,4 , and $10 \mathrm{Gyr}$, from left to right, respectively.

bluer than the mean RGB color (by $\gtrsim 0.13$ mag: our systematic color uncertainties are $\lesssim 0.1 \mathrm{mag}$ ), although a $Z=0.001$, 10 Gyr model is consistent with the blue edge of the observed RGB. Presumably a 10 Gyr model with $Z \sim 0.002$ would also be consistent with the data, while perhaps providing a better match to the observed CMD slope. We conclude that, if the bulk of the RGB stars are old ( $>10 \mathrm{Gyr}$ ), the metallicity range is roughly $Z=0.001-0.004$, while for younger ages the metallicity range shifts to higher values.

These results are comparable to the RGB analysis of the main body of NGC 4449 by A+08 (their Figure 17; see also Ryś et al. 2011). Therefore, both the main galaxy and the stream contain similar old, intermediate-metallicity populations, although the main galaxy also contains very young stars, as well as more metal-poor stars as inferred from its globular clusters (Strader et al. 2012).

We provide a preliminary overview of spatial stellar population variations by splitting the RGBs into color-based subpopulations, using the $4 \mathrm{Gyr}, Z=0.004$ model as a boundary. We then create stellar-density maps as before, for the two subpopulations separately. Using blue and green color coding to represent the subpopulations left and right of the model boundary, we show the results in Figure 2(c). The stream's bright parts have no visible RGB color gradient and have an overall color similar to the main galaxy's halo at radii of $\sim 3-5 \mathrm{kpc}$. Both the stream's faint-loop continuation and the halo at $\sim 5-10 \mathrm{kpc}$ are redder, implying older or more metal-rich stars.

\section{STELLAR AND DYNAMICAL MASS}

We now proceed to estimate the luminosities and masses of NGC 4449 and its stream. For NGC 4449, it is straightforward to add up the SDSS pixel fluxes inside the "optical radius" $\left(\mu_{r}=25 \mathrm{mag} \operatorname{arcsec}^{-2}\right)$. We find an extinction-corrected $M_{r}=-17.8$. For the stream, we use the SDSS-calibrated
BBRO image, integrating the flux within the faintest isophote that closes without including the main galaxy, which corresponds to $\mu_{g}=26.75 \mathrm{mag} \operatorname{arcsec}^{-2}$ and a stream semimajor axis distance of $3 \mathrm{kpc}$. We find a stream magnitude of $M_{r}=-13.5$, which is comparable to the brightest Local Group dSphs, Fornax, and And VII. Such galaxies have typical projected half-light radii of $\sim 0.4-1.0 \mathrm{kpc}$ (Brodie et al. 2011), which is consistent with the stream's $\sim 0.8 \mathrm{kpc}$ half-width.

For both galaxies, these luminosity estimates are lower limits since they do not include potential extended low-surfacebrightness features. The implied luminosity ratio is $\sim 1: 50$.

To calculate stellar masses $M_{\star}$, we use two different approaches, adopting a Chabrier (2003) initial mass function (IMF; final mass, including stellar remnants). The first is based on the integrated optical colors, following the relations between color and stellar mass-to-light ratio $\left(\Upsilon_{\star}\right)$ from Zibetti et al. (2009, Table B1). This Letter also introduced a technique for mapping out local $\Upsilon_{\star}$ and $M_{\star}$ variations pixel by pixel, which we apply to NGC 4449 , and after integrating, find a total $M_{\star}=7.46 \times 10^{8} M_{\odot}$. For the stream, we assume a global color corresponding to the central SDSS measurement, $g-r=0.45 \pm 0.1$. We find a stream mass of $M_{\star}=1.5_{-0.6}^{+0.8} \times 10^{7} M_{\odot}$, implying a stellar-mass ratio between stream and host of $\sim 1: 50$, for any uniform IMF. ${ }^{17}$

The second mass-estimation approach uses the CMD, comparing observed star counts to predicted numbers from a stellar populations model. For the stream, we use the I-band stellar luminosity function near the TRGB and normalize it to Monte Carlo simulations drawn assuming $Z=0.001-0.004$, and ages 2-10 Gyr. We obtain $M_{\star} \sim(2-5.5) \times 10^{7} M_{\odot}$ for the stream (a lower limit because of incompleteness), which agrees with the color-based results. For the main galaxy, a similar approach was followed by McQuinn et al. (2010), whose results imply $M_{\star}=(1.2 \pm 0.2) \times 10^{9} M_{\odot}$. This mass is somewhat higher than by using colors, but the CMD-based stellar-mass ratio turns out to be similar, $\sim 1: 40$.

Remarkably, the mass of NGC 4449's stellar halo is similar to the stream's mass: based on the RGB counts of Ryś et al. (2011) and their normalization to $K$-band surface-brightness photometry, we estimate $M_{\star} \sim 2 \times 10^{7} M_{\odot}$ for the halo between projected radii of 5-10 kpc. This halo could have therefore been built up directly by one or a few past accretion events similar to the present-day stream.

We next consider the dynamical masses of the host galaxy and its stream, including DM. The quantity that is arguably the most relevant to the current stream-galaxy interaction is the dynamical mass ratio within the interaction region: the $\sim 14 \mathrm{kpc}$ galactocentric radius. Based on the $\mathrm{H}$ I gas kinematics, we estimate an inclination-corrected circular velocity of $v_{\mathrm{c}} \simeq$ $62 \mathrm{~km} \mathrm{~s}^{-1}$ at this radius (Bajaja et al. 1994; Hunter et al. 2002), which means a dynamical mass for the main galaxy of $M_{\text {dyn }}(r<15 \mathrm{kpc}) \simeq 1.1 \times 10^{10} M_{\odot}$. The H I gas mass is $\sim 10^{9} M_{\odot}$ (Hunter et al. 1998), so this region is DM dominated. Note that the $v_{\mathrm{c}}$ and $M_{\star}$ values together suggest that NGC 4449 is intermediate in mass to the LMC and Small Magellanic Cloud (SMC; cf. Besla et al. 2010).

For the stream mass, we have no direct measurements, and instead turn to a plausibility argument based on Local Group dSphs, where the brightest cases have estimated circular

\footnotetext{
17 The similarity of the luminosity and mass ratios implies that the luminosity-weighted estimate of $\Upsilon_{\star}$ for NGC 4449 happened to turn out the same as inferred for the stream.
} 
velocities of $v_{\mathrm{c}} \sim 15-20 \mathrm{~km} \mathrm{~s}^{-1}$ on $\sim 1-3 \mathrm{kpc}$ scales (Walker \& Peñarrubia 2011; Boylan-Kolchin et al. 2012). Then if we assume the $v_{\mathrm{c}}$ values for both stream and main galaxy are fairly constant with radius, the ratio of $v_{\mathrm{c}}^{2}$ yields the dynamical mass ratio. This ratio is $\sim 1: 20-1: 10$, and thus the stream may be significantly perturbing the main galaxy.

A final metric is the ratio of total (virial) halo masses $M_{\mathrm{vir}}$, which are not directly measurable but may be inferred on a statistical basis, assuming a $\Lambda \mathrm{CDM}$ framework. In this context, it is well established that the total mass-to-light ratios of dwarf galaxies increase dramatically at lower luminosities. Current estimates of $M_{\star}-M_{\text {vir }}$ and luminosity $-M_{\text {vir }}$ relations (Moster et al. 2010; Tollerud et al. 2011) would imply $M_{\text {vir }} \sim$ (1-5) $\times 10^{11} M_{\odot}$ for NGC 4449, and a pre-infall mass of $\sim(1-10) \times 10^{10} M_{\odot}$ for the stream progenitor-which although very uncertain, plausibly implies an initial virial mass ratio of $\sim 1: 10-1: 5$.

Thus what appears to be a very minor merger in visible light may actually be closer to a major merger when including DM. Such an extreme circumstance could be compared with models of satellite disruption and potentially discriminate between $\Lambda \mathrm{CDM}$ and alternative theories (McGaugh \& Wolf 2010).

\section{DISCUSSION}

We have detected and analyzed a stellar tidal stream in the halo of NGC 4449, which we interpret as the ongoing disruption of a dSph galaxy by a larger dwarf (an LMC/SMC analogue ${ }^{18}$ ). This appears to be the lowest-mass primary galaxy with a verified stellar stream.

We suggest some implications for galaxy evolution. It has been proposed that dSph's orbiting massive galaxies such as the Milky Way were "pre-processed" from gas-rich dwarfs by tidal effects within dwarf-galaxy groups (D’Onghia et al. 2009). We may be witnessing such a transformation in-action, with the H I streams surrounding NGC 4449 representing additional tidal debris.

We also suspect it is not just a coincidence that such a novel stream was found first around one of the most intensely starforming nearby galaxies. The accretion event may well be the starburst trigger. The period of elevated star formation appears to have started $\sim 0.5$ Gyr ago (McQuinn et al. 2010), which is suggestively similar to the stream's $\sim 1-2$ Gyr orbital period ${ }^{19}$ (and to any process that is linked to the dynamical time on $\sim 30 \mathrm{kpc}$ scales).

Are such accretion events frequent among other dwarf galaxies in recent epochs? We suspect that exact analogues to this stream are not very common, or they would have been noticed already in DSS/SDSS images. However, if the stream had been only a bit fainter, more diffuse, or at a larger radius, it could have been missed, and thus there may be many more dwarf-hosted stellar streams awaiting detection.

In theory, the history of DM halo assembly should be fairly scale free, and $\sim 1: 10$ mergers are expected to be the most generally dominant contributors to mass growth (Stewart et al. 2008). It is also increasingly recognized that such relatively minor mergers can have important effects on the larger galaxies, such as inciting global disk instabilities (Purcell et al. 2011).

\footnotetext{
18 The LMC and SMC may also have a history of interaction, with a stellar-mass ratio of $\sim 1: 15$ (Besla et al. 2010).

19 Given a projected apocentric radius of $R_{\mathrm{a}}=13 \mathrm{kpc}$, a circular orbit provides a lower limit for the period of $T=2 \pi R_{\mathrm{a}} / v_{\mathrm{c}} \quad 1.3 \mathrm{Gyr}$.
}

If streams as in NGC 4449 are common in dwarfs, they reignite classic ideas about galaxy interactions triggering starbursts. Given the high rates of star formation in dwarf galaxies, it is natural to ask if satellites are responsible. Surveys along these lines have produced mixed results (Noeske et al. 2001; Brosch et al. 2004; Li et al. 2008), but until now, low-surfacebrightness objects such as dSphs would have been missed.

Regardless of the implications for starbursts, dSph accretion appears to be an increasingly viable avenue for direct assembly of dwarf galaxies' stellar halos-as witnessed by NGC 4449, and by Fornax, which shows traces of swallowing an even smaller dSph (Coleman et al. 2005). Future observational determinations of dwarf stream frequency in combination with theoretical models may provide clues to the general substructure problem.

We thank Jay Strader for a preview of his paper, and James Bullock, Pavel Kroupa, Jorge Peñarrubia, Monica Tosi, and the referee for comments. Based on data collected at the Subaru Telescope (operated by the National Astronomical Observatory of Japan), via Gemini Observatory time exchange (GN-2010B-C-204). F.A. received partial financial support from ASI, through contracts COFIS ASI-INAF I/016/07/0 and I/ 009/10/0. The Dark Cosmology Centre is funded by the Danish National Research Foundation. Work was supported by the National Science Foundation (Grants AST-0808099, AST0909237, AST-1109878, Graduate Research Fellowship), by NASA/Spitzer grant JPL-1310512, and by the UCSC-UARC Aligned Research Program.

\section{REFERENCES}

Annibali, F., Aloisi, A., Mack, J., et al. 2008, AJ, 135, 1900 (A+08)

Annibali, F., Tosi, M., Aloisi, A., van der Marel, R. P., \& Martinez-Delgado, D. 2012, ApJ, 745, L1

Arp, H. 1966, ApJS, 14, 1

Bajaja, E., Huchtmeier, W. K., \& Klein, U. 1994, A\&A, 285, 385

Besla, G., Kallivayalil, N., Hernquist, L., et al. 2010, ApJ, 721, L97

Boylan-Kolchin, M., Bullock, J. S., \& Kaplinghat, M. 2012, MNRAS, submitted (arXiv:1111.2048)

Brodie, J. P., Romanowsky, A. J., Strader, J., \& Forbes, D. A. 2011, AJ, 142, 199

Brosch, N., Almoznino, E., \& Heller, A. B. 2004, MNRAS, 349, 357

Chabrier, G. 2003, PASP, 115, 763

Cioni, M.-R. L., van der Marel, R. P., Loup, C., \& Habing, H. J. 2000, A\&A, 359,601

Coleman, M. G., Da Costa, G. S., Bland-Hawthorn, J., \& Freeman, K. C. 2005, AJ, 129, 1443

Cooper, A. P., Cole, S., Frenk, C. S., et al. 2010, MNRAS, 406, 744

D’Onghia, E., Besla, G., Cox, T. J., \& Hernquist, L. 2009, Nature, 460, 605

Ferrero, I., Abadi, M. G., Navarro, J. F., Sales, L. V., \& Gurovich, S. 2012, MNRAS, submitted (arXiv:1111.6609)

Girardi, L., Bertelli, G., Bressan, A., et al. 2002, A\&A, 391, 195

Hartmann, L. W., Geller, M. J., \& Huchra, J. P. 1986, AJ, 92, 1278

Huchtmeier, W. K., Karachentsev, I. D., \& Karachentseva, V. E. 2009, A\&A, 506, 677

Hunter, D. A., Rubin, V. C., Swaters, R. A., Sparke, L. S., \& Levine, S. E. 2002, ApJ, 580, 194

Hunter, D. A., van Woerden, H., \& Gallagher, J. S. 1999, AJ, 118, 2184

Hunter, D. A., Wilcots, E. M., van Woerden, H., Gallagher, J. S., \& Kohle, S. 1998, ApJ, 495, L47

Karachentsev, I. D., Karachentseva, V. E., \& Huchtmeier, W. K. 2007, Astron. Lett., 33, 512

Li, C., Kauffmann, G., Heckman, T. M., Jing, Y. P., \& White, S. D. M. 2008, MNRAS, 385, 1903

Lovell, M., Eke, V., Frenk, C., et al. 2012, MNRAS, 420, 2318

Marigo, P., Girardi, L., \& Chiosi, C. 2003, A\&A, 403, 225

Martínez-Delgado, D., Alonso-García, J., Aparicio, A., \& Gómez-Flechoso, M. A. 2001, ApJ, 549, L63

Martínez-Delgado, D., Gabany, R. J., Crawford, K., et al. 2010, AJ, 140, 962 
Martínez-Delgado, D., Peñarrubia, J., Gabany, R. J., et al. 2008, ApJ, 689, 184 Martínez-Delgado, D., Pohlen, M., Gabany, R. J., et al. 2009, ApJ, 692, 955

McConnachie, A. W., Irwin, M. J., Ibata, R. A., et al. 2009, Nature, 461, 66

McGaugh, S. S., \& Wolf, J. 2010, ApJ, 722, 248

McQuinn, K. B. W., Skillman, E. D., Cannon, J. M., et al. 2010, ApJ, 724, 49

Miyazaki, S., Komiyama, Y., Sekiguchi, M., et al. 2002, PASJ, 54, 833

Mo, H., van den Bosch, F. C., \& White, S. 2010, Galaxy Formation and Evolution (Cambridge: Cambridge Univ. Press)

Moster, B. P., Somerville, R. S., Maulbetsch, C., et al. 2010, ApJ, 710, 903

Noeske, K. G., Iglesias-Páramo, J., Vílchez, J. M., Papaderos, P., \& Fricke, K. J. 2001, A\&A, 371, 806

Ouchi, M., Shimasaku, K., Okamura, S., et al. 2004, ApJ, 611, 660

Purcell, C. W., Bullock, J. S., Tollerud, E. J., Rocha, M., \& Chakrabarti, S. 2011, Nature, 477, 301
Ryś, A., Grocholski, A. J., van der Marel, R. P., Aloisi, A., \& Annibali, F. 2011, A\&A, 530, A23

Schlegel, D. J., Finkbeiner, D. P., \& Davis, M. 1998, ApJ, 500, 525

Schweizer, F., \& Seitzer, P. 1988, ApJ, 328, 88

Stetson, P. B. 1987, PASP, 99, 191

Stewart, K. R., Bullock, J. S., Wechsler, R. H., Maller, A. H., \& Zentner, A. R. 2008, ApJ, 683, 597

Stinson, G. S., Dalcanton, J. J., Quinn, T., et al. 2009, MNRAS, 395, 1455

Strader, J., Seth, A. C., \& Caldwell, N. 2012, AJ, 143, 52

Tollerud, E. J., Bullock, J. S., Graves, G. J., \& Wolf, J. 2011, ApJ, 726, 108

Tolstoy, E., Hill, V., \& Tosi, M. 2009, ARA\&A, 47, 371

Walker, M. G., \& Peñarrubia, J. 2011, ApJ, 742, 20

Weisz, D. R., Dalcanton, J. J., Williams, B. F., et al. 2011, ApJ, 739, 5

Zibetti, S., Charlot, S., \& Rix, H. 2009, MNRAS, 400, 1181 